\title{
Sixth Sense Technology: A Variant for Upcoming Technologies
}

\author{
Devyani Agarwal \\ Student \\ Amity School of Engineering \\ and Technology \\ Amity University, Noida, India
}

\author{
Lakshay Malhotra \\ Student \\ Amity School of Engineering \\ and Technology \\ Amity University, Noida, India
}

\author{
Arunima Jaiswal \\ Asst. Professor \\ Amity University, Noida, India
}

\begin{abstract}
'Sixth sense' is a way of opening up to the vast world which cannot be identified by five senses. Mr. Steve Mann in 1990 started working on this idea which led to the innovation of 'Sixth sense technology' using a Telepointer, a wearable device for the experience of visuals on real world objects. His wok was then carried on by a younger lad, Pranav Mistry.

Sixth sense device is based on gesture interactions and its interface, a kind of device which doesn't need to carry any big attribute like a camera or watch. Six sense technology is a new $\&$ interesting stream of technology, which can be the most approachable as it is all about interacting with the digiworld directly. The device uses mirror, camera, pocket projector, smart phone \& color markers to identify any object or some view and then it imparts it over the real world.
\end{abstract}

\section{Keywords}

Sixth Sense, Gesture, Technology, Augmented Reality, Radio Frequency Identification, Computer Vision, Kinect.

\section{INTRODUCTION}

We come across some object, some person or some area, and so the tendency to use our five natured sense organs which include, two eyes, two ears, a nose, and a tongue; mind and soul to attain information about it; this knowledge relates in helping us chose the right and proper actions and take the correct path. The use of phones and tablets, which is, basically, minimization of social devices, keeps user $24 \times 7$ linked to the electronically linked social world. But we still can't find any link between our electrical devices and our daily chorus intercommunications with the physical, outside the technology, world. That is, the vice versa cannot be seen. Data is put over a sheet of paper traditionally or scientifically on a display screen or our phones.

Sixth sense technology covers this void, calling in untouchable, electrical information out into the real world, and giving users the allowance to communicate with this source through normal daily hand movements, making the entire approachable and touchable world your computer and does not confine information digitally.

SixthSense device is an apparel, "gesture based" device which progresses the real world with electronical information and this access is also given by it, by making people use their hand movements as an interface between the usage and device.

Mr. Pranav Mistry, a student persuing his $\mathrm{PhD}$ in the Fluid Interfaces Group at the MIT Media Lab is the developer of the device. A graduate student with the Fluid Interfaces Group at MIT, he created Very big start with his creation of SixthSense Device. His inspiration, as his words, were the movies "Minority Report" \& "Robocop" which gave him an idea for creating his own innovation part of the world which won't be under the actions of digital devices like computers, information or cyborgs, but an enjoyable world where healthy environment is found, a place where real world makes use of digital world, not its contrary, which is seen these days. [8]

\section{A BRIEF- SIXTH SENSE TECHNOLOGY}

'SixthSense' is a type of apparel, gestural interface that progresses the real world around users with the electronic based information and lets us use usual actions and hand movements to communicate with that information and data. Sixth Sense is scientifically, Extra Sensory Perception (in short ESP). ESP is basically sensing with the mind, without any recognisation by the five physical senses. It focusses on the past relations to present, having no relation with the to-be-actions. Sixth Sense mainly aims at more integrating online information and technology into the conscious world. By allowing information to the users for the decision making, by making means of five gifted senses, it proudly gives users a high technological sixth sense.

\section{WHY BUILT SIXTH SENSE TECHNOLOGY}

Humans are in habit of deciding after getting inputs from the five senses. These are his dependable organs. But it is well seen that this dependency gives him wrong results sometimes as it is not sufficient enough for the efficient decision making. But in this time with the help of Internet we collect a huge number of information which guides in making a good decision over a fraction of second. This six sense technology gives us the freedom of interacting with digital world with hand gestures .This technology has a good scope in the field of Artificial Intelligence. This methodology can also help in making of bots like humans.

\section{SIXTH SENSE PROTOTYPE}

The prototype of the device consists of three parts

- $\quad$ pocket projector,

- mirror component and,

- Camera component.

The hardware constituents are linked in a medallion like way where the camera and mobile feature is linked to the user's pocket.

The Sixth Sense Technology can be considered to be as a fusion of the mobile and adjoining computer. It works in a way where it is abided over the neck of the user and then the micro projector starts its work by projecting the result. This result to a movement wherein the user's body parts behave like the organs of a computer system, that is, users behave like the computer itself in which your limbs are doing the work of a keyboard and mouse.

A mobile, accessible to the World Wide Web was attached to a projector (powered by a battery) and a general webcam, 
associated with an attached mirror. The cost of the whole setting was lesser than $\$ 350$, and it allows users to view information from his phone onto any surface namely, walls, even the body of another person or your hand. Mistry wore the device, using a string around his neck. He colored his four fingers with red, yellow, green and blue Magic Marker caps which helped the camera distinguish the fingers and recognize his hand gestures with the software that Mistry had created. [8]

\section{BASIC REQUIREMENTS}

\subsection{Software}

The six sense technology software will be free sources, as the item related to it doesn't need any advancement or user interfaces. But it has many complexes, yet a very tightly tied coding inside the device to ensure if this software is set free from corruption. The sixth sense prototype runs on windows operating system with the major part of the software coded in languages like $\mathrm{C \#}$ and $\mathrm{C}++$. The upcoming newer versions of software will also include a mobile version for the users in future.

\subsection{Hardware}

\section{- Camera [13]}

Job is to seize images of the real world products in physical view \& correlates with movements of user's hands. It transfers data to mobile phone for further processing. It also infact, act as a digieye which connects to the virtual world of digitalization.

- Mobile component [13]

This part of the arrangement functions like Mobile telephone inside user's jeans via which data and voice is broadcasted and reserved via data packs, wifi or any other mobile web sources. An escorting Smartphone runs the prototype arrangement, and controls the networking to World Wide Web. Processing of the video data is done on this web enabled mobile network. Other software goes through the Web (virtual world) and interprets the hand gestures going on in the real world.

- $\quad$ Camera component [13]

Here computer vision algorithm plays its role as the webcam attached to the system tracks and records the user's gestures of hands and link them to perform operations or tasks. Smart phone receives data because of it. The camera in a way, behaves like a digital eye where it focusses on objects, users want to. The job is to keep a note of the gestural movement of user's thumb and fingers so that it can trace it over. The objects are noticed by this component, around users instantly, with the micro projector showing the information anywhere near user (which he wants), including the object itself or your hand with its movement.

\section{- Projector [13]}

Reciprocation and distribution with a purpose of sharing is carried out by the projector. It encapsulates a 3hour long working battery inside it. The projector showcases the virtual world on surfaces like walls and floor tiles, or any other physical surrounding objects around us which can be used as interfaces. Such a way projector enables merging of the real world into a new, real, physical manner. By the time we are sensing that object, at the same time projecting information onto that real world object. This data will look like it is already a part of the object. Small LED projector is placed in such a manner that it

Showcase data sent from your mobile telephone on whatever surface in view which maybe a part of a daily routine or ceiling, or even a person.

\section{- $\quad$ Colored Marker [13]}

These are marked with four colors at the ends of user's fingers. With the help of red, green, blue and yellow markers, the webcam focusses on hand movements and projects it on the virtual data. This gestural movement is then interpreted by the inside prototype which gives the instructions to the device as on how the application has to be run and outcome.

\section{WORKING OF DEVICE}

Multiple technologies namely, frequency identification, gesture recognition, image processing, etc. are used to function the device. Currently the evolving product for the market purpose isn't launched but the prototype adjoining is made for it. The best part of this sixth sense prototype is that it is made using very general and easily findable equipments like pocket projector, mobile components, a camera component, color markers and a mirror.

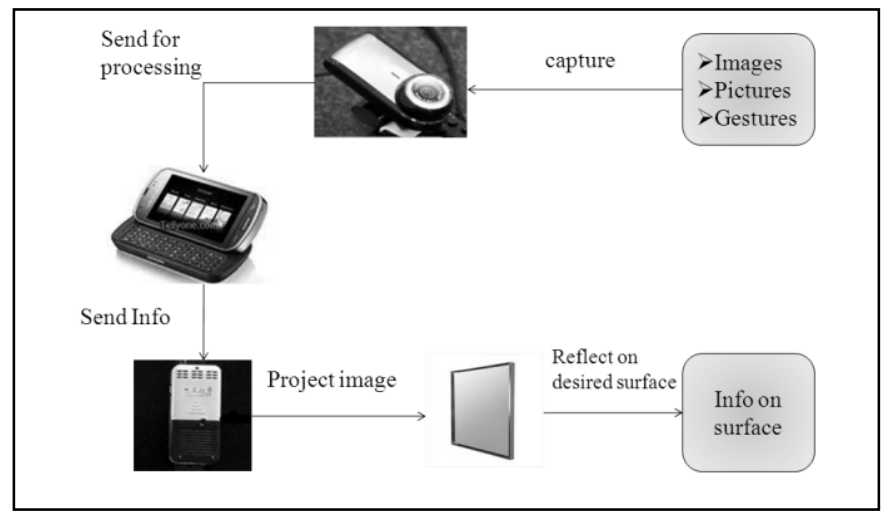

Fig 1: Working of device [3]

i. The projector provides the entire world on our screen by projecting virtual information on any flat surface which is comfortable to be used like, walls or fridges.

ii. The motions of users are captured by the camera component when the four colored fingers move over the object which has the virtual data.

iii. Noticing actions by making use of "Computer Vision Technique". The markers are used as visual tracking, fiducially.

iv. The inbuilt programme software's job is to convert these motions into gestures which are recognizable by the computing language so that we get the desirable outcome.

v. The projector projects the information feeded due to movements and gestures.

vi. The mirror does its task by showcasing the images projected by the projector. For a compact behavior, it is aligned in down direction. In such a way, pictures can be formed at the feet of user.

vii. The whole setting is structured in the form of a medallion.

viii. The prototype settings are cheap, yet the devices costs around $\$ 350$ because of expensive projector. [13]

ix. Its working is almost similar to a touch screen smartphone with entire world as its screen. 


\section{RELATED TECHNOLOGIES}

\subsection{Augmented Reality [2]}

Augmented reality (AR) can be defined as, a view of real or virtual observations of a physical part of world environment whose constituents are augmented (expanded) by digital imagery which is media generated. This is associated with a much common idea, that is, "mediated reality" where a live show of reality is improved (perhaps made to shrink instead) by a computing device. This augmentation usually takes place in actual-time and in linguistic state of affairs with components of the environment.

Sixth sense technology uses this concept of Augmented Reality to overlay virtual data on the real world. Using AR technology like adding computer visionary and products identification, users get compatible and virtually accessible information to their physical world. Artificial information about the surroundings and the objects within it can be saved and processed as another data surface primaraly over the physical world view.

AR consists of four main hardware components, namely,

- Track

- Display

- Input device

- $\quad$ Computing device

Smart phones can be a great potential platform as it may be a medley of powerful CPUs, cameras, accelerometers, GPSs and solid state compasses which are often present in their modems.

There are three main display techniques for AR:

1. Head Mounted Displays [2]

HMD is a technique which enables formation of visuals that are physically or virtually recorded (graphics) over the user's view of the sphere. They are categorized as- Optical see-Through and Video see-through.

\section{Handheld displays [12]}

This technique of AR uses any computing device which can display results and can be held by hand, no big arrangements required. It superimposes graphical data to real objects using Video see through method.

\section{Spatial Displays [2]}

Rather than having people wear or carry the display devices like the above, HMDs and handheld devices under AR; Spatial Augmented Reality (SAR) employs digital activators for displaying the visual information over the physical elements.

The Modern mobile AR systems use one or more than one of the below mentioned tracking technologies:

- Digital cameras or other optical sensing devices [13]

- $\quad$ RFID [1]

- Wireless sensors etc.

The levels of accuracy and clarity of each of these technologies are however different. They vary in a sense that in what way the head of user is positioned for detection via device. The augmentation takes place after detecting the posture, otherwise it won't function.

One of the socially convenient ideas of $\mathrm{AR}$ is that, it helps the disabled.

Its main advantage is that it is compatible with mobiles which are not really seen in other techniques.

\subsection{Gesture Recognition [2-3, 9-10]}

'The interpretation of human gestures and movements using logical algorithms and tables' is the main idea behind Gesture
Recognition [2]. Gestures can be made by any body part but usually are made by face and hands. But the most values recognitions for computing use are mainly two, Hand recognition (finger movements) and emotion recognition. It can also be called sign language.

Gestures may be made by hands itself or also by any object. This includes Freeing of things, way we wave, or action, sign, ward off, and in a way, making use of more usual sign languages for disabled. When related to things, we get a wide range of gestures that are very much in daily use, which includes, pointing at like pointers, motioning or touching things, altering shape of object, activisation of daily uses such as controls, accesors and handlers.

This type of technology gives world a way to recognize human actions and body language foreplaying on a routine basis, so inventing a relationship among the computer and the human with the help of gestures is the ideal condition for interaction, but it is still limited as majority of input takes place by mouse or keyboard. By Gesture recognition, an interface is evolved between humans and machines naturally as there is no mechanical device involved.

Gestures can be in the way like syntactic gestures which are underneath categorized according to their functions-

1) Symbolic Gestures [2,10]

Those gestures which have a proper meaning individually; For example, 'OK'.

2) Deictic Gestures [2,10]

Gestures which are used for pinpointing or directing a user towards some object or task. It is mainly used in $\mathrm{HCl}$.

3) Iconic Gestures [2,10]

These gestures are used to depict the physical appearance of objects. These gestures are observed when he hear people saying something like, "The rollercoaster went like this", while moving their hand across the sky like the flight path of the rollercoaster.

4) Pantomimic Gestures [2,10]

These gestures are different with iconic gestures in a way that these are typically used in showing the use of movement of some non-visual tool or thing in the speaker's hand. When a person actions "I turned the cycle hard to the left", while enacting the action of turning a two wheeler using his two hands, users use pantomimic gestures.

The main idea behind Gesture Recognition is that it can point towards the computer easily while enacting a computer cursor. It makes the existence of keyboards and mouse arbitrary as it may take their place in future. Computer visionary and Image processing are the two techniques under it. [9-10]

The current research is going on computer vision field which captures user's movements and humanly poses by camera component which is connected to the device.

\subsection{Computer Vision Based Algorithm [4-7]}

It is based on a Visionary with scientific and technical approach towards machines that vision. By the scientific aspect, computer vision is concerned with the theory behind artificial systems that extract information from images. The visual data can take many forms, such as video maker sequence, views from several cameras, or multi-dimensional data observed and noted from a medical scanner.

Computer vision studies the various programs and methodologies implied behind artificial gesture recognition systems. The software tracks the user's gestures using computervision based algorithms. In actual, the inverse of computer graphics is computer vision in some ways. Where computer 
graphics pictures image data or the video from some 3 dimensional model, computer vision does the vice versa, it often produces 3D models from image data. There is also a trend towards a combination of the two disciplines, e.g augmented reality.

The three fields which are most closely related to computer visionary are
a. Image processing, [11]
b. Image analysis and [11]
c. Machine vision. [11]

Image processing and image analysis focus on x-y axes images, how to transform one image to another. Computer vision focuses upon the features of the 3D object or scene, e.g., how to reconstruct structure or other information about the $3 \mathrm{D}$ scene from one or several images.

Machine vision focus on applying, that is, mainly in manufacturing, e.g., vision based robots and systems for vision based inspection or measurement.

\subsection{Technologies that uses Sixth Sense Prototype}

Sixth Sense Technology is found to be taking another approach for computing and this way make our lives more natural, interactive and intuitive when it comes to digital part. Pick up a packet of Waffles and the device will present whether it suits your health and diet. Such kind of technology is widely used by Radio Frequency Identification, Kinect and PS3 and Whirlpool washing machine or even Active app of TOI.

\subsubsection{Radio frequency identification [1]}

RFID is a non-contact wireless connection with the Radio Frequencies for transferring data using cracking of bar codes method to track items. Sixth sense is actually a phase of RFID.

It tracks the events, objects, actions and movements of users and then makes use of Radio electromagnetic frequencies.

Radio Frequency Identification is basically a method of electronic tagging technology which grants disclosure \& discovering of objects and thus the various parts and techniques they are adjoined with.

Sixth sense device, developed by Pranav Mistry, is a device which can be worn on user's wrist (for comfort) which then works as a touch screen for the modernized apps. [13]

Examples of RFID as a Sixth sense technology are Calendar system and online activity presence which tracks users. [1] For such tasks, RFID takes help from other enterprises too. Soon in future, a time will come when RFID tags are used for cheaper online sale of objects and products for user routine, rather for employees having laptops and mobile phones tagged with inexpensive passive tags, with a good number of RFID readers in their offices.

Sixth Sense consolidates coding that have a set of similar tags axiomatically inferring a scope of data based on the observations and readings collected. This technique is capable of recognizing the tags associated with people or objects in a way to gain knowledge, data, information related of identy of users, ownership criteria of products, work nature and environment of offices (private versus conference room) and comprehending other such inferences.

The comprehensive detailed information is feeded into high level databases by joining all data from these diverse sources, that is, the raw events and then they are categorized to level of work, doers and objects which is then converted into specialized databases for workplace. Such databases are constructed into SQL queries on oracle.

Sixth Sense comes into force when the action takes place like when the user comes in contact with the object, for instance, when he picks his cellphone. It is a stage where the programming algorithm makes the interferences made axiomatically accessible to application through a diverse pact of APIs. Few of some aptitudes of researcher's prototyped applications using these APIs which also includes heightened alarm system, a misplaced object alert system and diverse illustration of video with real world happenings.

\subsubsection{Whirlpool Washing machine [15]}

Washing machine by Whirlpool, model AWOE 8758 is exceptional and impressive main leader which incorporated the emerging Sixth sense technology in its 2009 White Washing machine batch. It was a package of the machines working under the influence of Sixth sense technique that gave new optimization of sources and also led to a great evolvement in the social aspects of human resources which are

$$
\begin{array}{ll}
\text { - } & \text { Water } \\
\text { - } & \text { Time } \\
\text { Energy }
\end{array}
$$

Washing machine work is to clean the stains and removes dirt from clothes and apparels. Sixth sense washing machine helped by detecting huge stains on clothes and then dexterously helped in removing such discolorations, adjust wash impact and detect permanent black spots. This enhanced the washing mechanism and several other customizations.

New generation Sixth sense washing machine helped in protecting environment by helping users in using less of energy which reduced their energy bills and saved energy. They are constructed in a way that it intelligently and smartly behaves as energy efficient tool to better the performance as per user requirements. There are three keys on which these washing machine work, namely,

$$
\begin{array}{ll}
\text { - } & \text { Sense } \\
\text { - } & \text { Adaption } \\
\text { - Control }
\end{array}
$$

These ensure that the machine achieve the optimal performance every time it is run.

The objective is to save energy, time and water and it succeeds by saving up to $50 \%$ of it during its run. These smart washing machines adjust the size of load during its run and then manage the amount of water, time and energy required to complete the cycle. Some of its model also makes a note of amount of detergent to be used and whether the detergent is getting over dosed; saving the amount of detergent required to run the cycle. There are tumble dryers installed in the machines to monitor the humidity inside the cleaning cycle. It also adjusts the drying time accordingly. This is enhancing of laundry system.

\subsubsection{Sixth sense Kinect}

With the launch of Microsoft Kinect in 2010, this technology gained a huge upheaval in the technological market. This device is installed in Xbox360, quite famous among gamers, and senses the actions and motion of gamers. These motions are projected in the game. This gives a real time experience to game lovers.

\section{FUNCTIONS OF SIXTHSENSE DEVICE}

\subsection{From Digital World to Physical World}

Digital world represents the camera and Physical world represents human's actions and behavior pattern. Sixth sense device senses this behavior like drawing, reading, and writing and bring it into the digital world. For example, while reading any magazine or novel, if the user holds it book in front of the camera, the device will detect the book name, publication, price, availability areas and various other credentials involved. Also, it may give links to the book review by critics and readers and read out the review for users if they want. This enhances listening 
experience. Similarly, while going through newspaper headlines, the user may be able to search the topic on World Wide Web and get a deep insight into it. He may also watch it by being linking to its visual part. For example, the much used ACTIVE app of TOI. Furthermore, if one is listening to some music on radio and cannot detect which song is it, he/she can feed it into the microphone of the device and it will provide the user with its information, For example, Shazam app on Play store of Android phones.

Usually, the Paint tool on computer is tough to use, so one may draw it physically and save it on PC.

Finally, entertainment is another Wide function. As a projector can put the digital world on sheets of paper, and the camera captures the movement of fingers, the same way users can play the vehicle racing game as well as other sorts of games on a piece of paper.

\subsection{From Physical world to Digital World}

According to this method, first is to capture using camera. Now it is not necessary to click pictures with a digicam or dslr. Instead, one can use his fingers to make a specific movement or behavior and consequently the device will get to know what the user demands and then complete his demand.

Most exciting experience can be that the device moves over a block of physical text to the blank paper directly. Moreover, users can edit the text on their notebooks and copies and visuals on the paper and press the print command digitally which gives out the data on the paper.

Text or pictures captured can be sent from the paper directly into the $\mathrm{PC}$, the reverse process too.

\section{APPLICATIONS}

The greatest benefit of the device is the availability of wide variety of application in it they are -

1. Calling feature

2. Make up a map

3. Check the time

4. Interactive media reading venture

5. Picturing and Drawing any app

6. Enhancing and whirling features

7. Products and appliances information

8. Booked information

9. Get airways updates

10. Recording information of people

11. Capturing pictures

12. Checking of email

\section{CONCLUSION}

Sixth Sense is such an emerging highly advanced technology that it will lead to a great advancement in our daily life.

1. Use of papers and pens will decrease.

2. Online shopping may become easy if, Virtual changing room, where one can give his body measurements and try clothes on a virtual copy of his image, is created by web developers.

3. It can become a fifth sense for handicapped people. [2]

4. Future enhancements of Infrastructure.

5. New way of teaching in Education System.

6. Videos can be widely annotated by physical events side by side.

\section{ISSUES AND CHALLENGES FACED BY TECHNOLOGY}

One of the major concerns of this technology is the high level of engineering and coding required which requires high back-end system. Secondly, this will cost above $\$ 350$, as told by Mistry. [13] Also, during projection, there is no privacy as anyone can see it from anywhere. Vision of customer can also be damaged. So, health can get affected.

\section{REFERENCES}

[1] SixthSense: RFID-based Enterprise Intelligence by Lenin Ravindranath, Venkata N. Padmanabhan, Piyush Agrawal, Microsoft Research India, Indian Institute of Technology Kanpur.

[2] SIXTH SENSE TECHNOLOGY by Arjun K R, 2010 DSpace Cusat.

[3] THE SIXTH SENSE TECHNOLOGY by Mandar Ghate, Padmabhushan Vasantdada Patil Pratisthans College Of Engineering, Mumbai.

[4] Handbook of Mathematical Models in Computer Vision by Nikos Paragios, Yunmei Chen, Olivier Faugeras (2005) ISBN 0-387-26371-3.

[5] Processing: An Algorithmic Approach Using Java by Wilhelm Burger and Mark J. Burge (2007) ISBN 1846283795 and ISBN 3540309403.

[6] Computer Vision - Principles and Practice. by Pedram Azad, Tilo Gockel, Rüdiger Dillmann (2008) International Media BV. ISBN 0905705718

[7] Computer Vision - Three-Dimensional Data from Images by Reinhard Klette, Karsten Schluens and Andreas Koschan (1998) Singapore ISBN 981-3083-71-9.

[8] Sixth Sense Technology by Abhinav Sharma, Mukesh Agarwal, Anima Sharma, Sachin Gupta, International Journal IJRITCC Volume 1, Issue 4, 277-282.

[9] Gesture Based Interface Using Motion and Image Comparison by ShanyJophin, Sheethal M.S, Priya Philip, T M Bhruguram, IJAIT Vol. 2, No.3, June 2012.

[10] GESTURE BASED OPERATING SYSTEM CONTROL by Yuvraj V. Parkale, Electronics and Telecommunication Department College of Engineering, Malegaon (Bk), Maharashtra, India 2012 Second International Conference on Advanced Computing \& Communication Technologies.

[11] Vision based Analysis using Sixth Sense Technology by Swarali Narvekar and Manali Godse, International Journal of Computer Applications (0975 - 8887), International Conference and Workshop on Emerging Trends in Technology 2013.

[12] Real Time Hand Gesture Interface for Computing Environment by Shitalkumar Pawar and M.A. Sayyad, International Journal of Computer Applications (0975 8887)(ICRTET'2013).

[13] WUW - Wear Ur World - A Wearable Gestural Interface by Pranav Mistry, Pattie Maes and Liyan Chang, MIT Media Lab, ACM 978-1-60558-246-7/09/04.

[14] SixthSense - A Wearable Gestural Interface by P. Mistry, P. Maes, Proceedings of SIGGRAPH Asia 2009, Yokohama, Japan (2009). 
[15] How we look at the World Forever by Sixth Sense Technology by Ms.Uttama Suryavanshi, International Journal of Advanced Research in Computer Science and Software Engineering, vol. 3 Issue 11, November 2013.

[16] Sixthsense Technology: The New Digital Revolution by Chen Zhang, University of Sydney, 5PM Journal of Digital Research.

[17] International Journal of Advanced Research in Computer Science and Software Engg. 3(11), November 2013,pp.744-748 by Suryavanshi.
[18] Sixth Sense Technology Or Wuw (Wear Ur World) by Bathani, Raxa, Research Expo International Multidisciplinary Research Journal 2.2 (2012) : 236-243.

[19] Telepointer: Hands-free completely self-contained wearable visual augmented reality without headwear and without wearable visual augmented reality without headwear and without any infrastructural reliance by Steve Mann, University of Toronto, Dept. Electrical and Computer Engineering. 